\title{
Research on Life Extension Method of Transmission Line Intelligent Sensing System Based on Environmental Energy Harvesting
}

\author{
Wanli Sun $\left(\mathbb{D}\right.$, Yu Liu $\mathbb{D}^{\circ}$, Lei Ma $\mathbb{D}^{\circ}$, and Ruopu Zhang $\mathbb{C}$ \\ Department R\&D, Beijing SmartChip MicroElectronics Technology Company Limited, China \\ Correspondence should be addressed to Wanli Sun; sunwanli@sgitg.sgcc.com.cn
}

Received 27 January 2021; Revised 30 July 2021; Accepted 25 August 2021; Published 18 September 2021

Academic Editor: Changhui $\mathrm{Hu}$

Copyright (c) 2021 Wanli Sun et al. This is an open access article distributed under the Creative Commons Attribution License, which permits unrestricted use, distribution, and reproduction in any medium, provided the original work is properly cited.

\begin{abstract}
In this paper, we focus on building and researching the self-power sensor system based on the tiny energy harvesting technology which can be used in the scenario of ubiquitous power Internet of Things (UPIoT) and prove the possibility and efficiency for the life extension of transmission line intelligent sensing system. Large scale of sensor in the smart grid technology application, especially in high-voltage transmission lines, is not convenient; it is worth mentioning that most of all kinds of sensor node equipment using battery power make the life of the equipment or components limited by greatly. Therefore, low power consumption, long life, no battery dependence, free maintenance, and other requirements are increasingly important to solve the difficulties of deployment and maintenance. At the same time, the problem of the battery case electricity use with low efficiency and short life of sensor node has become the bottleneck of further widely deployed wireless sensor node equipment. The power collection technology based on environmental energy can effectively handle with the problems of energy collection efficiency and management that need to be urgently solved in new application scenarios such as zero-standby power consumption devices, remote active tags, battery-free telemetry and remote control, and ultralong life sensing system. By studying various kinds of environmental energy collection technologies and utilizing the conversion and management technologies of available weak environmental energy, such as solar energy and magnetic field energy, into electric energy, this paper establishes an energy conversion test system and configuration model and verifies the feasibility of the assumption of maintenance-free for the intelligent sensing system of transmission lines.
\end{abstract}

\section{Introduction of Transmission Line Intelligent Sensing System and Environmental Microenergy Collection Technology}

Smart grid sensing system of the transmission line, conductor temperature, and image conductor is a practical and monitoring system, such as dancing and overhead line transmission tower in the process of forming and ops bolt used in all kinds of intelligence, pressure, and settlement. A huge number of angle sensor mainly depends on the degree of intelligent degree of maintaining the stable working. Therefore, most wireless sensor network nodes are powered by disposable batteries. After being installed, it is very difficult or impossible to replace the equipment maintenance and battery under high voltage and live environment. As a result, energy supply and management of nodes have always been the focus of research in wireless sensor network technology. Although measures of optimizing network structure and reducing node power consumption can reduce energy consumption to a certain extent, they cannot fundamentally solve the problem of node energy depletion. However, the technology of available energy collection which can be collected continuously from the surrounding environment opens up a new direction for solving this problem $[1,2]$. At present, the energy collection technologies that have been 
applied in the field of power transmission line sensing mainly include solar energy harvesting and magnetic field harvesting.

1.1. Solar Energy. Solar energy extraction mainly converts light energy into electric energy through solar panels to supply power for sensors. Since the energy intensity of light energy varies greatly with time, solar panels are often combined with batteries to form solar power supply systems in practical applications [3]. The principle is shown in Figure 1. The solar power supply system is relatively mature, with high energy conversion efficiency.

The conversion efficiency of mass-produced photovoltaic cells can reach up to $18 \%$, and the energy density is about $3 \mathrm{~mW} / \mathrm{cm}^{2}$. However, the application effect is limited by natural conditions and battery life [4].

1.2. Magnetic Field Energy. Magnetic field energy harvesting technology mainly relies on closed coils to generate induced current by induction of the changing magnetic field, so as to realize the conversion of magnetic energy to electric energy [5]. Magnetic field energy capture technology can be divided into invasive and noninvasive according to the deployment mode of energy capture module. Invasive energy harvesting is similar to conventional current transformer (CT) (as shown in Figure 2).

By plugging into the transmission line or AC bus, it generates coupling effect to extract electricity. The coupling effect is good, the energy harvesting power is high, but the operability is poor, and the later maintenance cost is high [6]. Noninvasive energy extraction means that the energy extraction module is placed outside the energy-being extracted device, and energy extraction is carried out through external coupling effect. As shown in Figure 3, the coupling coefficient of noninvasive energy extraction and the power of energy extraction are lower than that of invasive energy extraction.

The installation of noninvasive energy extraction device does not destroy the structure of the original system and has good operability with low maintenance cost [7]. At present, the invasive magnetic field energy harvesting technology is relatively mature, which can realize the magnetic field energy harvesting under the load of $5 \mathrm{~A}$ and above lines, and the output power can reach watt level, which has been widely used in power system. Engineering application cases mainly include transmission line temperature sensor based on CT energy extraction, distribution line fault indicator, etc. However, the noninvasive energy capturing technology has few practical applications.

1.3. Laser Power. The main principle of the laser power supply mode $[8,9]$ is that the laser light source is received at the low potential side. After that, the energy is transmitted to the high potential side through the optical fiber. Then, the light energy is directly converted into electric energy by the photoelectric conversion device, and the stable power output is finally provided after passing through the DC-DC converter. Its advantage $[10,11]$ is that under relatively stable conditions, a relatively stable power supply voltage can be obtained after conversion; the power supply has small ripple,

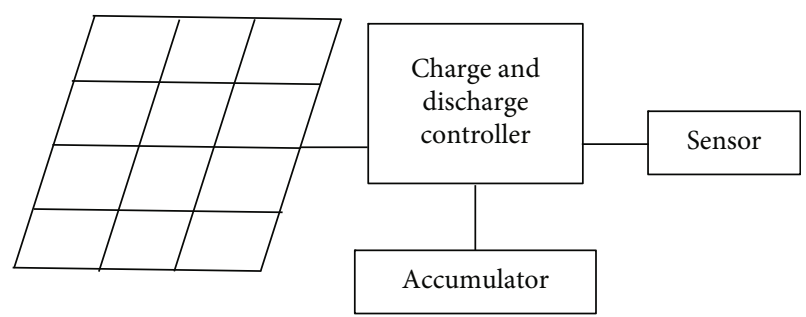

Figure 1: Schematic diagram of solar power supply system.

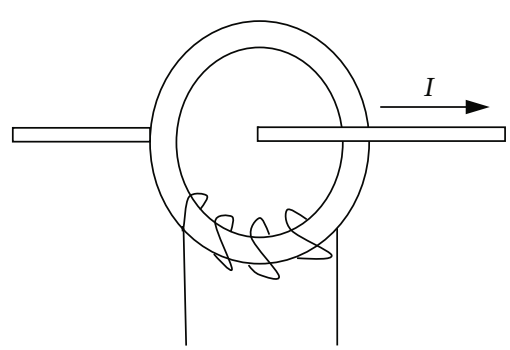

Figure 2: Invasive energy harvesting.

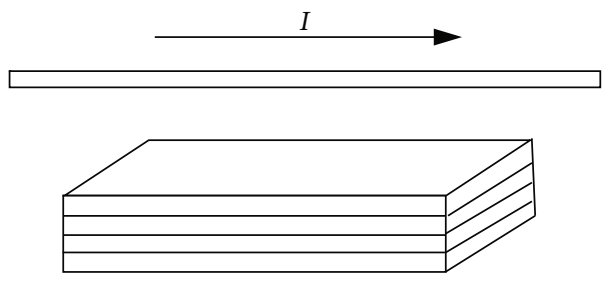

Figure 3: Noninvasive energy harvesting.

low noise, and is not easily affected by external factors. This power supply mode also has some disadvantages, such as low photocell conversion efficiency, limited laser output power, and limited power supply.

1.4. Microwave Power. Microwave power supply is a wireless power transmission method [12]. In the process of transmission, the energy loss of microwave is smaller than that of laser power supply, and the transmission energy is larger, which has the characteristics of simple and convenient implementation. However, there are also some shortcomings in the application, such as bringing some interference signals in the microwave transmission process to the normal operation of online equipment, especially in the data transmission and relay protection equipment action.

1.5. Voltage Transformer. The principle of voltage transformer power supply $[13,14]$ is the same as that of ordinary transformer. A special voltage transformer (PT) is adopted to transform the electric energy from high voltage to low voltage and provide to the electric equipment. It has a stable power output and easy access to electric energy points. The disadvantages are large volume, high cost, and inconvenient installation, which have great influence on operation safety.

Different energy harvesting technologies are suitable for different fields. In response to the needs of large-scale breeding farms for monitoring the ventilation status, a wind- 
TABLE 1: Characteristic of battery and supercapacitor.

\begin{tabular}{|c|c|c|}
\hline Characteristic & Battery & Supercapacitor \\
\hline Transformation & Chemical-electrical & Electrical \\
\hline Internal reaction & REDOX chemical reactions & Polarized electrolyte physical reactions \\
\hline $\begin{array}{l}\text { Process } \\
\text { reversibility }\end{array}$ & $\begin{array}{c}\text { Charge and discharge process reversible, energy } \\
\text { conversion loss }\end{array}$ & Charge and discharge process reversible \\
\hline $\begin{array}{l}\text { Charging and } \\
\text { discharging speed }\end{array}$ & $\begin{array}{l}\text { The general charge and discharge rate is } 1-5 \text { times, the } \\
\text { maximum discharge rate can be up to } 10 \text { times }\end{array}$ & $\begin{array}{l}\text { The higher the charging current, the faster the speed, } \\
\text { and within } 10 \text { seconds can reach } 95 \% \text { of the rated } \\
\text { capacity }\end{array}$ \\
\hline $\begin{array}{l}\text { Operating } \\
\text { temperature }\end{array}$ & $-25^{\circ} \mathrm{C}-+45^{\circ} \mathrm{C}$ & $-40^{\circ} \mathrm{C}-+70^{\circ} \mathrm{C}$ \\
\hline Energy density & High, $20-100 \mathrm{wh} / \mathrm{kg}$ & Low, 3-15 wh/kg \\
\hline $\begin{array}{l}\text { Charge-discharge } \\
\text { efficiency }\end{array}$ & $>95 \%$ & $>95 \%$ \\
\hline Cycle life & $5000-10000$ & $>100000$ \\
\hline $\begin{array}{l}\text { Environmental } \\
\text { protection }\end{array}$ & Potential pollution & Almost no chemical contamination \\
\hline Maintenance & Periodic replacement & Maintenance-free \\
\hline
\end{tabular}

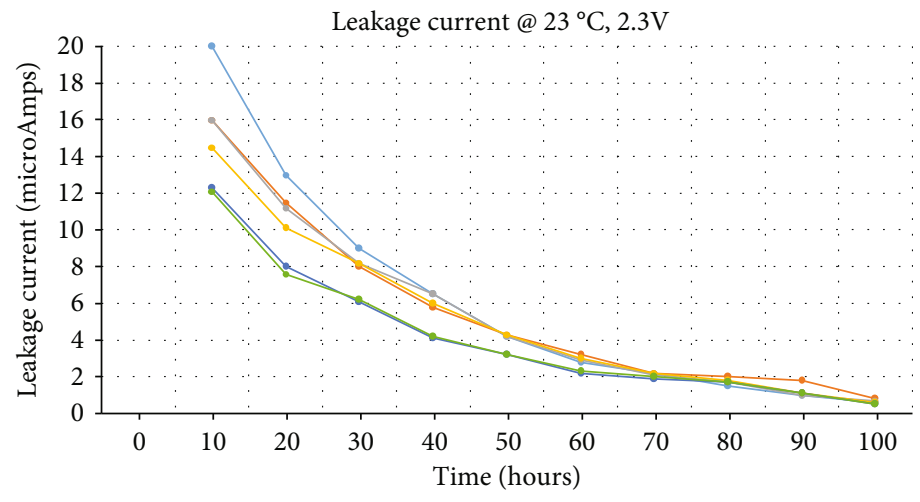

FIGURE 4: Discharge characteristics of supercapacitor samples.

induced vibration piezoelectric energy capture system can be designed to solve the problem of self-powered monitoring sensors [15]. The sensor self-powered system based on thermoelectric power generation can collect the energy of the temperature difference between the inside and outside of the ventilation pipe to supply power to the sensor monitoring system [16]. For outdoor IoT scenarios, solar energy can be used as an energy source, and the output characteristics of photovoltaic cells can be used for energy harvesting, and the voltage transmission ratio can be improved to realize the system self-powered [17].

\section{Selection of System Energy Storage Devices}

Rechargeable batteries are commonly used in wireless sensor systems for energy storage. Compared with standard capacitors and batteries, supercapacitors have many advantages, making them as ideal substitutes. These advantages include short charge and discharge times than rechargeable batteries, high efficiency which is up to $98 \%$, lower internal resistance, large power output, better thermal performance, and a better
TABle 2: Comparison of supercapacitor charging time.

\begin{tabular}{lccc}
\hline Super CAP & Super CAP-1 & Super CAP-2 & Super CAP-3 \\
\hline Parameter & & & \\
Beginning voltage & $0.7 \mathrm{~V}$ & $0.7 \mathrm{~V}$ & $0.7 \mathrm{~V}$ \\
Full voltage & $4.48 \mathrm{~V}$ & $4.48 \mathrm{~V}$ & $4.48 \mathrm{~V}$ \\
Charging time & $11 \mathrm{mins}$ & $9 \mathrm{mins}$ & $7 \mathrm{mins}$ \\
\hline
\end{tabular}

TABLE 3: Comparison of supercapacitor leakage characteristic.

\begin{tabular}{lccc}
\hline Super CAP & Super CAP-1 & Super CAP-2 & Super CAP-3 \\
\hline Discharge test result & & & \\
& $1.5 \mathrm{~F}, 5 \mathrm{~V}$ & $1.5 \mathrm{~F}, 5 \mathrm{~V}$ & $1.2 \mathrm{~F}, 5 \mathrm{~V}$ \\
T0 & 4.48 & 4.48 & 4.48 \\
T0+2 hours & 3.47 & 3.384 & 4.196 \\
T0+4 hours & 3.214 & 3.203 & 4.119 \\
T0+6 hours & 3.019 & 3.052 & 4.042 \\
\hline
\end{tabular}




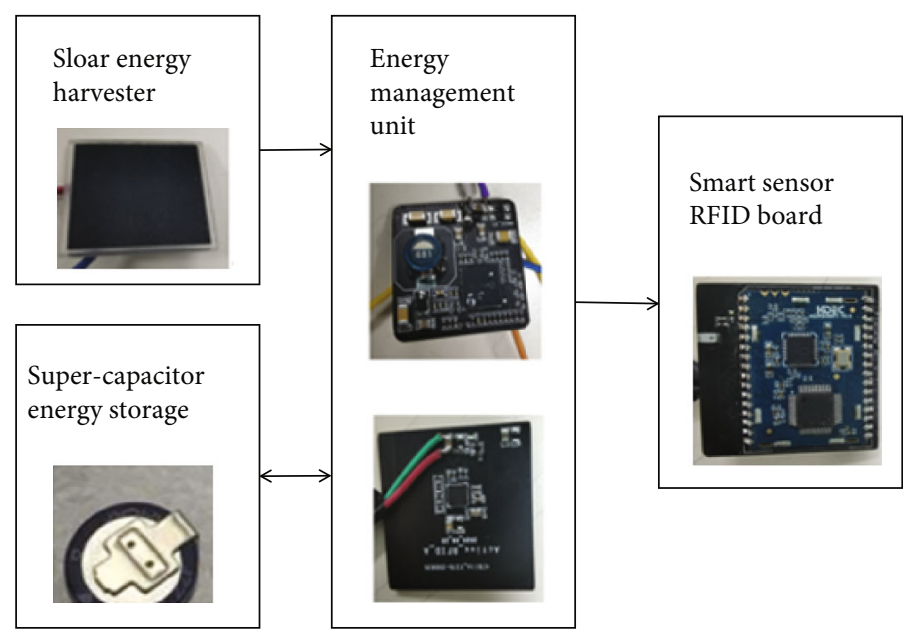

Figure 5: Sensor system diagram.

safety margin than batteries and standard capacitors. More differences between these two kinds of energy storage devices can be found in Table 1 .

Supercapacitor is an electrochemical component that uses polarized electrolyte to store energy. It can be used as a power source with special performance between traditional capacitor and battery. Moreover, the energy storage process is reversible, and it can be charged and discharged repeatedly for hundreds of thousands of times. Its outstanding advantages are high power density, short charge and discharge time, long cycle life, and wide operating temperature range. The selection of supercapacitors, power requirements, discharge time, and system voltage variation play a decisive role. There are two factors that will cause the voltage drop of the supercapacitor. One factor is the energy released by the supercapacitor; the other factor is the internal resistance of the supercapacitor. In the very fast pulse, the internal resistance part is the main factor. In contrast, in the long process of discharging, the capacitive part is the main part.

Supercapacitors have recommended operating voltage or optimum operating voltage based on the maximum working time of the capacitor at its highest set temperature. If the applied voltage is higher than the recommended voltage, the life of the capacitor will be shortened. If the overvoltage lasts for a long time, the electrolyte inside the capacitor will decompose and form a gas. When the pressure of the gas increases gradually, the safety hole of the capacitor will break. A short period of overvoltage is tolerable for a capacitor.

When a supercapacitor is discharged, it discharges according to a slope curve. When an application specifies the capacitance and internal resistance requirements of the capacitor, it is most important to understand the influence of resistance and capacitance on discharge characteristics. Resistance is the most important factor in pulse applications, and capacity is the most important factor in small current applications. The calculation formula is as follows:

$$
V=I\left(R+\frac{T}{C}\right)
$$

TABLE 4: Sensor system configuration model.

\begin{tabular}{lcc}
\hline Load parameter & & Marks \\
\hline Vin (V) & 3 & System operation voltage \\
T0 (s) & 1800.73 & Operation period \\
T1 (s) & 0.73 & Operation time \\
Isys (A) & 0.015 & Operation current \\
Isleep (A) & 0.000014 & System-free current \\
Iavg (A) & 0.00001 & System average current \\
$c$ (W/S) & 0.001217 & System average power \\
\hline
\end{tabular}

$V$ is the starting work voltage, and as the difference between the working voltage, $I$ is the discharge current, $R$ is the DC resistance, $T$ is the discharge time, and $C$ is the capacitance in pulse application. Because the current is very big, in order to reduce the voltage drop, the low resistance (ESR) of the supercapacitor ( $R$ value) is chosen; in small current applications, the large capacity of supercapacitor ( $C$ value) needs to be chosen in order to reduce the voltage drop.

Self-discharge and the leakage are essentially the same, in view of the supercapacitor structure, equivalent to inside the capacitance between the positive and negative has a high resistance current path; it means at the time of capacitor charging, there will be an additional current at the same time. When a capacitor is in charge, we can regard this current as leakage current. When the charging voltage is removed and the capacitor is not connected to the load, the current causes the capacitor to be in the discharge state. At this point, the current is regarded as the selfdischarge current.

In order to make the measurement of leakage current or discharge current more accurate, we chose the general supercapacitor samples which discharge characteristics are shown in Figure 4. The capacitor must be continuously charged for more than 72 hours to ensure the leakage slope tends to be stable. The leakage is determined by the structure of the capacitor. A supercapacitor is a parallel connection of 
TABLE 5: Calculation results under the condition of $2 \mathrm{~mA}$ charging current.

\begin{tabular}{lcc}
\hline Vmax (V) & 4.8 & Maxim voltage of output \\
Vmin (V) & 1.8 & Minimum voltage of output \\
Ileakage (A) & 0.00001 & Leak current of supercapacitor \\
Icharge (A) & 0.002 & Charging current under indirect solar conditions \\
Tdis (hrs) & 72 & Discharge time (determined by requirement:3 days without solar) \\
Capacity (F) & 4 & Capacity of supercapacitor \\
Tcharge (hrs) & 1.65 & Charge time \\
\hline
\end{tabular}

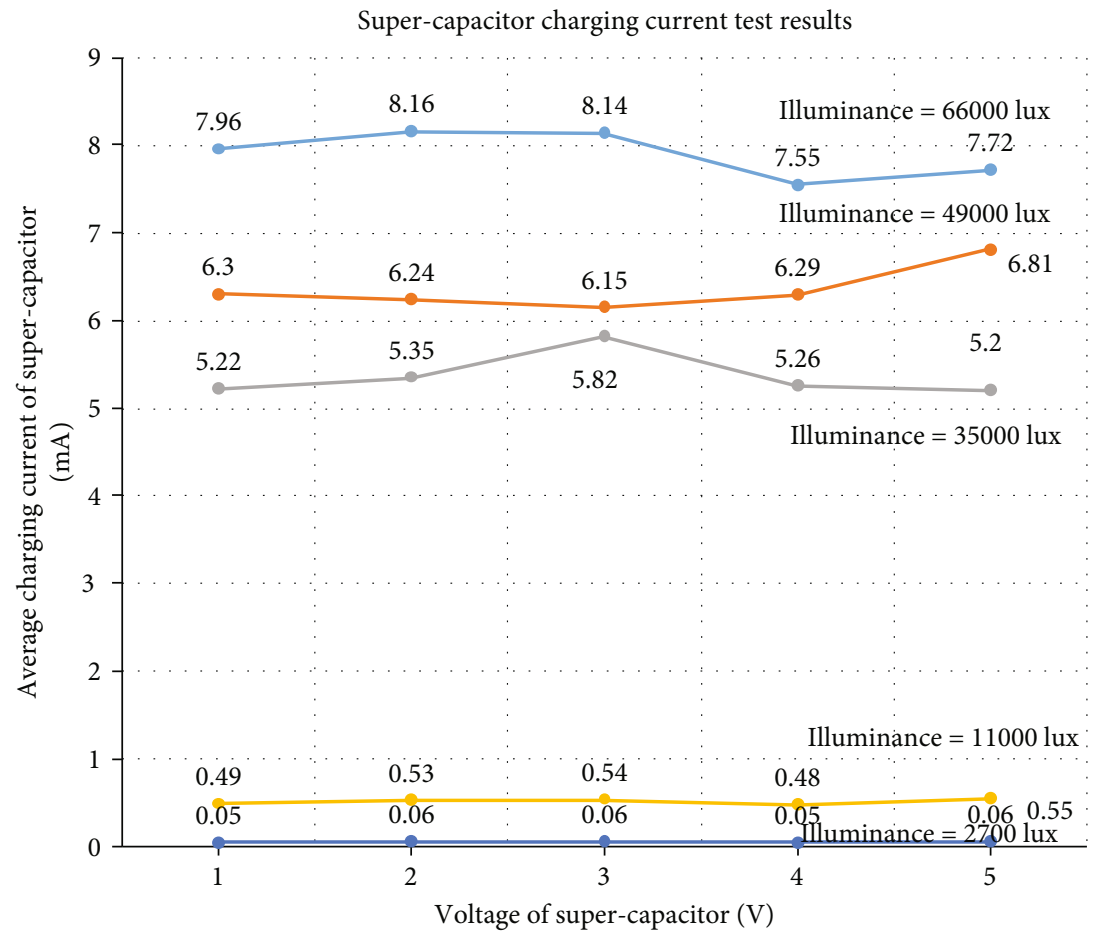

FIGURE 6: Supercapacitor charging current with the change of illuminance.

several supercapacitors with different internal resistances. When the supercapacitor is charged, the supercapacitor with low internal resistance charges fast, and the voltage can quickly rise to the value of the charging voltage. When the charging voltage of the supercapacitor is removed, if the high internal resistance supercapacitor is not fully charged, the low internal resistance supercapacitor starts to discharge to the parallel high internal resistance supercapacitor, so that the voltage across the capacitor will drop relatively fast. The capacitor is usually considered as a relatively large self-discharge. It must be noted that the larger the capacitance of the capacitor, the longer it takes for the capacitor to be fully charged.

In this paper, three types of supercapacitors are selected to test the actual charge-discharge effect. The system is set with an overvoltage protection of $4.5 \mathrm{~V}$ and a discharge threshold of $2.8 \mathrm{~V}$. The test results are as follows:

According to the test data shown in Tables 2 and 3, supercap-3 has excellent performance in terms of the overall charge-discharge effect and self-leakage; supercap-3 is selected as the energy storage element of the system.

\section{Verification of Self-Powered Sensor System Based on Microsolar Energy Extraction Sensor}

With the development of ubiquitous power Internet of Things, all kinds of wireless smart sensors are gradually widely used. In order to solve the problem of energy acquisition, support the long life cycle, and reduce the cost of manual maintenance of power system, this system focuses on the investigation of the energy demand of passive sensors commonly used in power system and selected the representative smart sensor RFID system as the research object to verify the feasibility.

The system converts solar energy into electric energy through a $2.5 \mathrm{~cm} * 2.5 \mathrm{~cm}$ solar panel suitable for small 


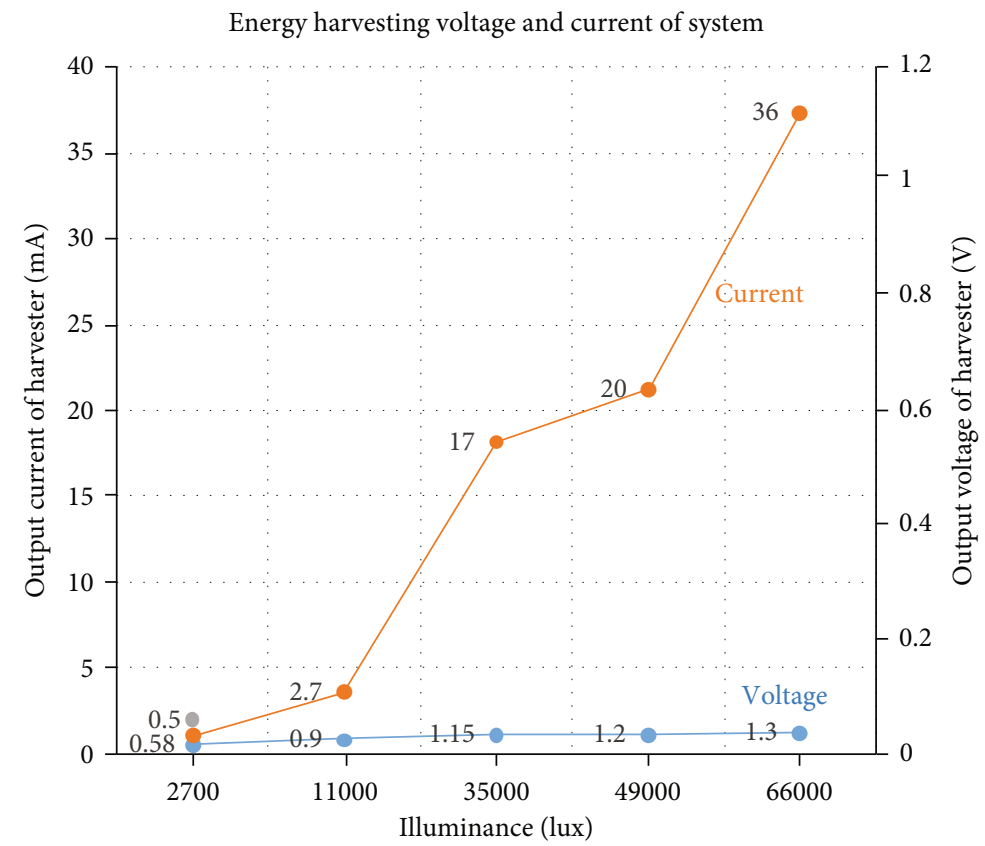

FIGURE 7: Energy harvesting voltage and current from solar panel.

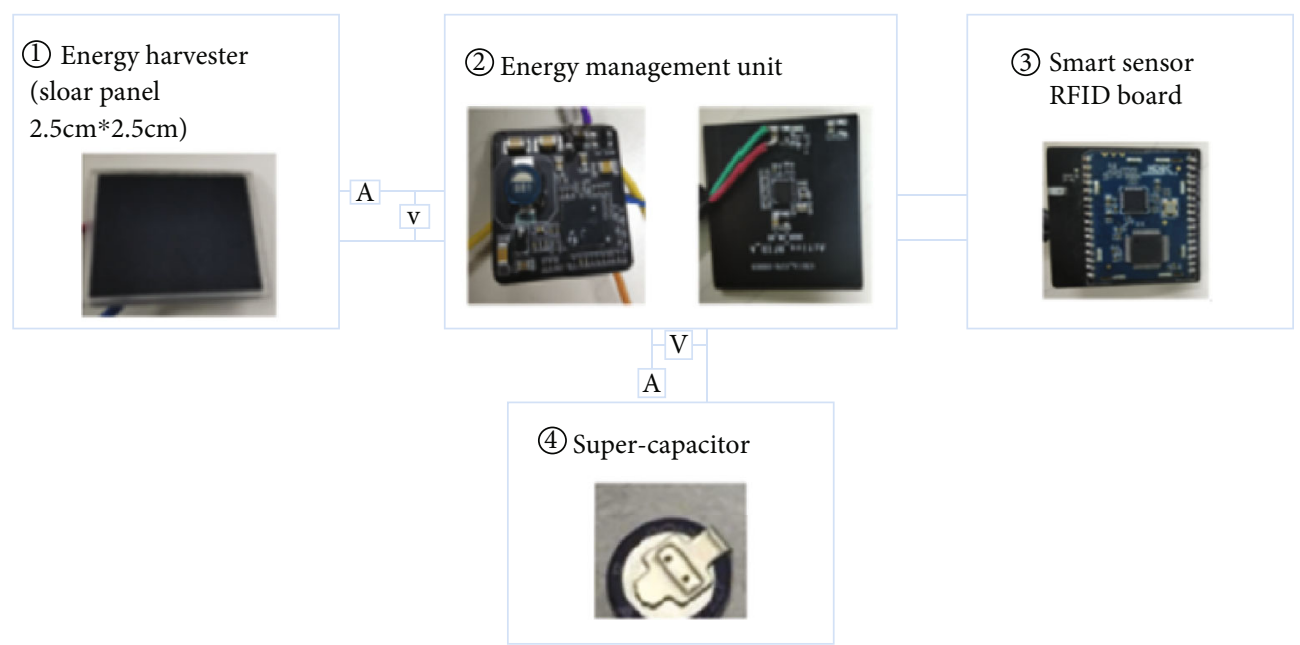

Figure 8: Experimental test platform.

sensor nodes and then converts nanoelectrical energy (only a few hundred milliwatt) into electric energy that can be used for smart sensor RFID system controllers through a power management chip with ultralow voltage start-up characteristics. Current system uses small solar panels as energy input, according to the previous section of the contrast of characteristics between supercapacitors and the ordinary rechargeable batteries; environmental temperature change is big. Considering the characteristics of large environmental temperature variation, different system load energy consumption, and high real-time charging frequency of environmental energy in practical applications, this paper uses the supercapacitor as the energy storage element and connects the back end to a real pressure data reporting sensor.
Based on the above analysis, we built a sensor system that can take a long life without maintenance as shown in Figure 5. The sensing system consists of solar panel energy harvester, microenergy management unit, smart sensor RFID board, and supercapacitor. The system emits a $15 \mathrm{~mA}$ current signal every 30 minutes which continues $730 \mathrm{~ms}$. System's free current is $14 \mu \mathrm{A}$. Supercapacitor leakage current is $10 \mu \mathrm{A}$.

3.1. System Configuration Model. The sensor node power supply system is based on ambient energy collection research output model, through calculation of the load power system based on the need to meet the continuous running time. The configurations of supercapacitors and solar 
panels are required to guide the system hardware architecture of the subsequent application design. At the same time, it can be used to evaluate the efficiency of the docking of other energy extraction devices.

Firstly, the average power of the system is calculated through the load power consumption, and the results are shown in Table 4.

$$
P(\text { average power })=\frac{[(\text { Vin } \times \text { Isys } \times T 1)+(\text { Vin } \times \text { Isleep } \times T 3)]}{T 0},
$$

$\operatorname{Iavg}($ average current $)=\frac{[(\text { Isys } \times T 1)+(\text { Isleep } \times T 3)]}{T 0}$.

According to the system configuration in the previous section, the following data parameters can be obtained. According to the above formula, the average power consumption of the system under the configuration condition of the system is $1.217 \mathrm{~mW}$, which can be used to calculate the supercapacitor configuration of the subsequent system under the condition of no light for 3 days.

The requirements of working voltage threshold, load current, the self-leakage, and working time of the supercapacitor are important, and the factors also were analyzed in the previous section; the capacitance required by the system can be calculated according to the average leakage of current, and the formula is shown as follows:

$$
\begin{aligned}
\operatorname{Capacity}(F) & =(V \max +V \min ) \times I \times \frac{t}{V \max ^{2}-V \min ^{2}} \\
& =\frac{(V \max +V \min ) \times \text { Ileakage } \times 60 \times 60}{(V \max 2-V \min 2)},
\end{aligned}
$$

Tcharge $($ Charge time $)=\frac{[C \times(\mathrm{Vmax}-\mathrm{Vmin}) / \mathrm{Icharge}]}{3600}$.

Table 5 shows the calculation results according to the charging efficiency after the output current of solar panels under solar indirect conditions converted by the power management system, and the charging current is $2 \mathrm{~mA}$.

Finally, the result obtained through the data model simulation is that when the ultralow power management system is configured with the upper limit of the charging voltage of the supercapacitor is $4.8 \mathrm{~V}$, and the discharge is as low as $1.8 \mathrm{~V}$; the size of the supercapacitor required for the system to maintain no light for 3 days is 4 Farad.

3.2. Experimental Test Platform and Results. In order to evaluate the system feasibility, we developed an experimental test platform, as shown in Figure 5, which is comprised of energy harvester, energy management element, supercapacitor, and smart sensor RFID board as load. One supercapacitor was chosen as an electric storage element to be evaluated considering the situation for nonlight. Two ammeters (A)

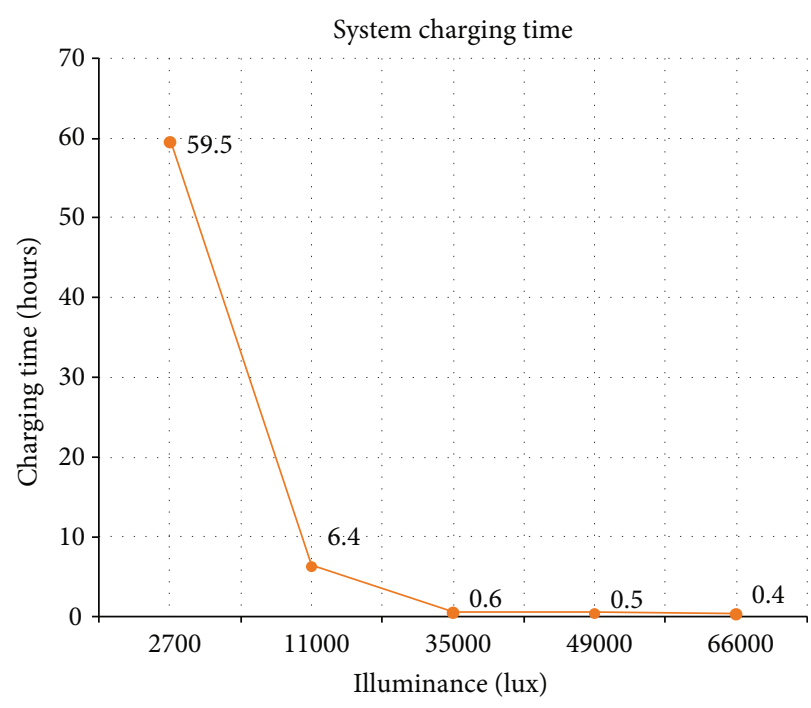

FIGURE 9: System charging time.

and voltmeters (V) are used for measurements of current and voltage, respectively.

It is very meaningful to test system charging current under the real different lighting conditions. Figure 6 shows the test results of supercapacitor charging current under the change of illuminance, the stronger the light, the greater the charging current. If the illuminance is 66000 lux, the charging current of system can reach $7.55 \mathrm{~mA}$ with the 4-Farad supercapacitor.

The energy provided by the energy harvester almost determines the available power of the system. Figure 7 shows the energy harvesting voltage and current test results.

To ensure the system normal operating, supercapacitor's voltage range should be $1.8 \mathrm{~V} \sim 4.8 \mathrm{~V}$; this experiment tested the charging time of supercapacitor from $1.8 \mathrm{~V} \sim 4.8 \mathrm{~V}$. As shown in Figure 8, the charging time is 0.42 hours when the system works under the condition of illuminance = 66000 lux which is shown in Figure 9. This light intensity is consistent with sunny day.

Considering the application model as shown in Table 4, the discharging time is tested under the condition of no input power. If we choose the 4-Farad supercapacitor, the system can work continuously for 73.3 hours without interruption (as shown in Figure 10).

As all the research above, the sensing system we built emits a $15 \mathrm{~mA}$ current signal every 30 minutes which continues $730 \mathrm{~ms}$. By calculation, the average current consumption of the system is $0.006 \mathrm{~mA}$. System's free current is $14 \mu \mathrm{A}$, and the supercapacitor leakage current is $10 \mu \mathrm{A}$. We can get that the total system power consumption is $30 \mu \mathrm{A}$. When the load voltage and charging voltage are the same, as long as the charging current of the supercapacitor is greater than $30 \mu \mathrm{A}$, it can ensure uninterrupted operation of the sensor node system. As shown in Figure 6, under the condition of illuminance greater than 11000 lux, the charging current is about $500 \mu \mathrm{A}$. Two hours of the same light intensity can meet the consumption of the system 24 hours a day. 


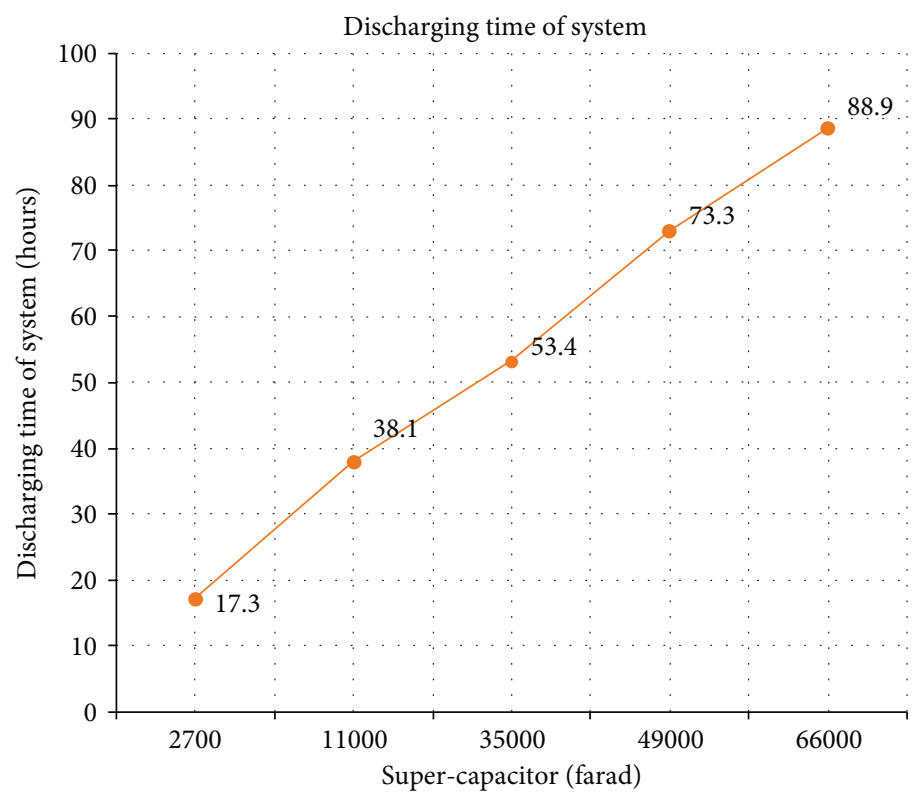

FIGURE 10: Discharging time of system test results, under the condition of no input power.

\section{Conclusions}

This work proposes a sensor micropower energy collection system and an effective detection method according to the energy utilization in the working environment of the electric energy sensor. The experimental results confirmed the feasibility of using environmental energy to supply power to the sensor system. The system can realize the self-powering of the low-power sensor nodes and ensure the long-term stable operation of the sensor node. By analyzing the current status of microenergy harvesting technology and the general characteristics of wireless sensors, a theoretical model of sensor energy supply-demand relationship is created. In practice, it is equipped with a microenergy harvesting management system model based on low-sensitivity sensor sensors. The actual test results demonstrate in theory and practice that the use of a microenergy harvester, a low-power ultralow voltage start-up power management chip, and a supercapacitor system architecture can provide the daily energy required by the wireless sensor system. In this way, small solid-state batteries can be added to prevent the system from inadequate energy supply accidents, extending the working life of the sensor and the maintenance-free period. So, the energy harvesting system solves the problem of uninterrupted self-supply of sensor nodes without nonrechargeable battery. The experimental results show that the self-powered system can solve the energy supply problem of low-power smart sensors, save manpower maintenance costs, use environmental energy to obtain energy, reduce environmental pollution, and improve the reliability of sensor detection devices. The experimental results also show that it is feasible to obtain energy from the natural environment to supply a low-power sensor system. At the same time, this solution would rely on and be restricted by the development of energy harvester technology, so we use the most mature energy harvesting equipment to do the research; in order to improve energy utilization efficiency, further research on energy harvesting technology and energy storage technology is needed, including energy harvesting materials, methods, and shapes.

The study also for the integration of intelligent sensor and transmission line tower label provides a possibility and can solve common problems of transmission tower label, because the passive label communication distance is very short; it must be hanged on the low part of the tower, but it is not convenient for human check. So, the minimized power system would make it possible to hang on the top of the tower; also, the combination of smart sensor and RFID system will further promote development of power transmission lines in intelligent monitoring scenarios of the system integration process. It provides important and reliable technical support for its intelligentization, miniaturization, and integration.

Self-powered sensors based on environmental energy harvesting have the advantages of long life and maintenance-free. This technology will become a hot spot and development trend in the research of low-power active systems in the future, especially for the use of energy resources in the working environment of electric power sensors under typical application scenarios of electric power. In order to solve the power supply problem of the power transmission and transformation status monitoring sensors, the abundant magnetic field energy around the transmission cable can be used to develop a self-powered system based on magnetic field energy for the transmission cable and converter valve application scenarios to improve the reliability and stability of the monitoring sensors. With the construction and development of ubiquitous power Internet of Things, wireless sensing technology has broad application prospects in the fields of power equipment status monitoring, power system fault diagnosis, and fault early warning and location of transmission lines. Transmission lines are 
an important part of the power grid. Regular inspections of transmission lines are the main means to ensure the normal operation of the power grid. Regular inspections waste manpower, financial resources, and material resources, so state inspections are used. As temperature sensor, humidity sensor, or strain clamp sensor that detect important states of transmission lines, its power supply cannot meet the long-term and reliable operation of the sensor. The multiparameter energy harvesting system can solve the power supply problem of a variety of wireless sensor devices.

\section{Data Availability}

The data used to support the findings of this study are included within the article.

\section{Conflicts of Interest}

There is no conflict of interest regarding the publication of this paper.

\section{Acknowledgments}

This study was funded by the project "Research on communication protocol of power wireless sensor network for power transmission and transformation state monitoring and development of prototype" which belongs to the Department R\&D, Beijing SmartChip MicroElectronics Technology Company Limited.

\section{References}

[1] Z. H. O. U. Feng, Z. H. O. U. Hui, and D. I. A. O. Yinglong, "Development of intelligent perception key technology in the ubiquitous Internet of Things in electricity," Proceedings of the CSEE, vol. 40, no. 1, pp. 70-82, 2020.

[2] J. I. A. N. G. Xiuchen, L. I. U. Yadong, and F. U. Xiaofei, “Construction ideas and development trends of transmission and distribution equipment of the ubiquitous power Internet of Things," High Voltage Engineering, vol. 45, no. 5, pp. 13451351, 2019.

[3] Z. H. U. Yongcan, H. U. A. N. G. Xinbo, and Z. H. A. N. G. Guanjun, "Application research of the power supply for transmission line on-line monitoring devices," High Voltage Apparatus, vol. 54, no. 7, pp. 231-236, 2018.

[4] Z. H. A. N. G. Min, Q. I. N. Yu, and W. A. N. G. Hongbin, "Design of power supply source of transmission line on-line monitoring device," Guangdong Electric Power, vol. 31, no. 10, pp. 94-100, 2018.

[5] G. U. O. Shen, W. A. N. G. Peng, and Z. H. A. N. G. Jichuan, "An overview of electromagnetic energy collection and storage technologies for a high voltage transmission system," Energy Storage Science and Technology, vol. 8, no. 1, pp. 32-46, 2019.

[6] P. E. N. G. Xiying, C. U. I. Dandan, and Z. H. A. O. Qiangsong, "Energy collection based power supply for monitoring equipment on transmission line," Mechanical Engineering \& Automation, vol. 2, pp. 192-194, 2017.

[7] L. I. Dong, "Study on magnetoelectric energy conversion technique used for space magnetic energy harvesting in substation," Electrotechnics Electric, vol. 6, pp. 10-15, 2017.
[8] C. Wang and B. S. Liyi, "Analysis and research on laser power supply condition of electronic current transformer under low temperature environment," High Voltage Apparatus, vol. 56, no. 5, pp. 0135-0142, 2020.

[9] Zhou, "A low current ripple power supply of laser diode for wireless power transfer," Chinese Science and Technology Papers, vol. 12, no. 11, pp. 1252-1256, 2017.

[10] Y. Shen, J.-m. Ge, R.-t. Liu, W. Fan, and Y.-X. Yang, "Optimization of optical current sensor based on rare-earth magnetooptical glass," Microwave and Optical Technology Letters, vol. 61, no. 2, pp. 490-497, 2019.

[11] P. Sarajcev and D. Jakus, "Optimal scheduling of power transformers preventive maintenance with Bayesian statistical learning and influence diagrams," Journal of Cleaner Production, vol. 258, p. 120850, 2020.

[12] A. V. Ubaychin, T. A. Uulu, and G. Zhuk, Microwave Radiometer for Sensor Systems with Self-Contained Power Supplies SJEM026022880037, Emerald, England, 2020.

[13] P. Bocui, "Research on high voltage side power supply scheme of active electronic voltage transformer," Mechanical and electrical engineering, vol. 29, no. 11, pp. 1323-1328, 2012.

[14] H. Rui, Research on Energy Management of Self-Powered Wireless Sensor System Based on Environmental Energy Harvesting, University of Electronic Science and Technology of China, 2019.

[15] W. Zhidong, F. Junlong, S. Peidong, F. Yuchen, W. Guangya, and P. Di, "Design method of self-powered sensor system based on piezoelectricity," Journal of Beijing Institute of Technology, vol. 40, no. 12, 2020.

[16] W. Zhidong, Z. Hongbin, F. Yuchen, C. Youjie, and B. Li, "Design of sensor self-powered system based on thermoelectric power generation," Laboratory research and exploration, vol. 39, no. 11, 2020.

[17] L. Shuiping and C. Jiahao, "Design of self-powered wireless sensor network nodes based on wind energy harvesting," Digital Manufacturing Science, vol. 18, no. 04, 2020. 